\title{
Surface pressure model for simple delta wings at high angles of attack
}

\author{
A A PASHILKAR* \\ Flight Mechanics \& Control Division, National Aerospace Laboratories, \\ Bangalore 560 017, India \\ e-mail: apash@css.cmmacs.ernet.in
}

MS received 21 December 2000; revised 27 March 2001

\begin{abstract}
A new aerodynamic modelling approach is proposed for the longitudinal static characteristics of a simple delta wing. It captures the static variation of normal force and pitching moment characteristics throughout the angle of attack range. The pressure model is based on parametrizing the surface pressure distribution on a simple delta wing. The model is then extended to a wing/body combination where body-alone data are also available. The model is shown to be simple and consistent with experimental data. The pressure model can be used as a first approximation for the load estimation on the delta wing at high angles of attack.
\end{abstract}

Keywords. Delta wing; surface pressure; aerodynamic modelling; high angle of attack.

\section{Introduction}

The drive to improve combat performance of fighter aircraft has invariably led to excursions into the high angle of attack regimes of flight. It has been shown that short excursions into these flight conditions result in significant improvements in tactical advantage over the adversary (Zagainov 1993). Clearly, for fighter aircraft, high angle of attack capability is desirable. However, at high angles of attack, the aerodynamics of delta wings is essentially nonlinear in nature. These nonlinear characteristics arise due to vortex flow and its breakdown over delta wings, which result in nonlinear longitudinal static characteristics (normal force and pitching moment). The static variation of aerodynamic coefficients obtained from the wind tunnel must be captured in an appropriate mathematical model. The aerodynamic model can then be used for predicting the flow field of the aircraft.

Understanding static data of a wing or wing/body configuration is an important first step in the development of models for the aerodynamics of complete aircraft at high angles of attack, for the following reasons. First, static data contain within them selves the information of significant changes in the flow physics over the wing. Understanding this aids in the development of simple model structures in agreement with the changes in the flow behaviour. Second, small amplitude unsteady aerodynamic characteristics are directly related to the static characteristics. Flight mechanics analysis is primarily concerned with the aerodynamic data composed of a combination of static and small amplitude forced oscillation data. Finally, 
large amplitude unsteady aerodynamics is also related to the nature of the static data. The static data are the limiting case of unsteady flow pattern as time tends to infinity (or at least a few times the characteristic time scale of the unsteady effects).

Many different approaches have been used to model the static aerodynamic characteristics of lifting bodies. One could broadly classify these approaches as a priori and a posteriori. In the former category would fall all those methods of aerodynamic model structure development obtained from the fundamental physical descriptions of the underlying aerodynamic phenomena. For example, computational fluid dynamic methods (Shamroth 1985; Tuncer et al 1989), the indicial response method (Tobak \& Schiff 1976), and axiomatic modelling approach (Hancock \& Lam 1987) fall in this category. The importance of these methods lies in their ability to bring to light the physical causes of aerodynamic characteristics. Such methods are characterized by high computational and modelling effort. On the other hand, the a posteriori methods require aerodynamic data of the aircraft in question either from the wind tunnel or from flight tests, for the development of the model structure. These methods include the semi-empirical methods (Lieshmann \& Beddoes 1989), the multipoint approach (Jaramillo 1994), the multivariate polynomial function approach (Morelli 1995), splines with limited support (Klein \& Batterson 1983), neural network models (Raol \& Jategaonkar 1995). Such methods can be adapted easily for purposes of parameter estimation. Yet another interesting approach is to couple some form of the aerodynamic model to the equations of motion (Myatt \& Arena 1998). Naturally, this involves more computations and is less amenable to parameter estimation for model improvement. The process may also involve undesirable $a d$ hoc 'tuning' of the aerodynamic model to match experimental data.

The various approaches discussed above can also be classified in terms of the fundamental assumptions they make about the model structure. On this basis, it is seen that the multivariate polynomial function approach, splines with limited support and neural network models are purely data reduction methods. They do not in any way make assumptions about the physical nature of the processes that they are representing. The only possible assumptions are of a mathematical nature regarding continuity etc. of the functions being represented. On the other end of the spectrum are the computational fluid dynamic methods, the indicial response method, and axiomatic modelling approach, which introduce assumptions, or models of the underlying fluid dynamic phenomena. In between are the semi-empirical methods and the multipoint approach. These methods introduce some of the physical models of the aerodynamic processes and develop a relatively simpler model structure that is suitable for analysis or simulation.

Although accurate simple prediction methods exist for aerodynamic characteristics of lifting bodies, e.g. Polhamus's (1966) suction analogy, they are limited by the onset of separated flows at high angles of attack. Traub (1997), has developed a prediction method for the longitudinal characteristics, which is based on the use of Polhamus suction analogy. Certain assumptions about the vortex lift behind the breakdown location are introduced in order to account for the phenomena of vortex breakdown. The results indicate that the method gives good predictive capability at low and moderate angles of attack, while the agreement between model and data is fair at high angles of attack. However, the parametrization is not sufficient to allow better fit to the data and thus the model cannot be used for improving the fit. It is also assumed that the spanwise pressure distribution is given by a particular function due to Purvis (1981).

This paper postulates a simple and physical model structure that captures the static variation of longitudinal aerodynamic characteristics with angle of attack. The modelling is based on parametrizing the surface pressure distribution. As opposed to a predictive model, the current 
model is parametric and hence more suited for estimation. The following criteria have been used as guides for model development.

(a) A physical as opposed to a purely mathematical model is desirable. Such a model results in parameters that can be checked for plausibility. It also gives insight into the fluid dynamics of the aircraft at high angles of attack.

(b) A simple model is certainly desirable from the point of view of analysis. All factors, which are clearly not important to the characterization, should be removed. Over-detailing can obscure the features of a model and in some cases introduce new behaviour not consistent with experiment. This must be avoided at all costs.

\section{Aerodynamic forces and moments}

In attached flow conditions for delta wings, theoretical estimates of the lift forces and moments using Polhamus leading-edge suction analogy show agreement with experimental values. When large-scale separation is present on an aerodynamic body, which is the case at high angles of attack, this theory cannot be applied. At a fundamental level, the forces and moments experienced by a body immersed in flow arise from the surface forces on the common boundary between the body and the surrounding fluid. These surface forces in turn can be divided into pressure forces normal to the body surface and shear forces parallel to it. It is well-known that for thin streamlined bodies, the normal force and pitching moment coefficients are almost entirely determined by the integral effect of the pressure distribution. This is true even when the body is at high angles of attack. The action of the shear forces is restricted to the boundary layer. These forces determine the total axial force, which is not critical to stability analysis. This observation is supported by the delta wing data of Wentz \& Kohlman (1968). It is clear that if the pressure distribution over lifting bodies is modelled in a suitable manner, a model structure can be obtained for the longitudinal characteristics throughout the angle of attack range. On the other hand, this demands that the qualitative changes in the pressure distribution parameters be known as functions of angle of attack.

The simplest way of developing a wing/body model is to apply the component build-up approach. Accordingly, a model for the delta wing longitudinal aerodynamic characteristics is developed. This is applied to wing/body data. The delta wing static aerodynamic model is based on characterization of the surface pressure.

\section{Experimental data}

Roos \& Kegelman (1990) have measured the surface pressure distributions at various spanwise and chordwise locations for a range of angles of attack for $60^{\circ}$ and $70^{\circ}$ delta wings. Zohar \& Er-El (1988) have also reported similar findings for $60^{\circ}$ and $75^{\circ}$ delta wings. Both the observations have been used to develop the model structure in this paper. The principle finding used from the experimental results is the pressure variation leeward of a delta wing.

The model structure based on the surface pressure distribution has been applied to the $60^{\circ}$ delta wing longitudinal aerodynamic data of Wentz \& Kohlman (1968). Also available in this work is the observed breakdown location of the leading edge vortices over the delta wings for a range of sweep angles. The normal force and pitching moment data reported in Zohar \& Er-El (1988) is not consistent with that in Wentz \& Kohlman (1968). This does not 


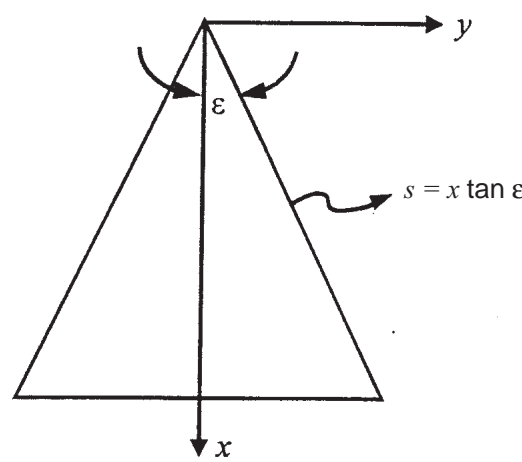

Figure 1. Coordinate system for the delta wing.

detract from the use of data from Zohar \& Er-El (1988) to develop the surface pressure model as the qualitative changes in the surface pressure model are independently confirmed by Roos \& Kegelman (1990).

Longitudinal experimental wing-body data for wing sweep angles of $50^{\circ}, 60^{\circ}$ and $70^{\circ}$ and for two bodies of slenderness ratios 7.8 and 9.8 were obtained from the work of Vishwanath \& Patil (1992). The data are in the form of $C_{z}$ and $C_{m}$ as functions of incidence at almost $0.25^{\circ}$ intervals. The leeward surface pressure distribution has also been reported for the wing/body combination of $60^{\circ}$ delta and $7.8 \mathrm{l} / \mathrm{D}$ body at fixed chordwise and spanwise location.

The axis convention adopted in this paper for delta wings is shown in figure 1 . The coefficients in this paper are non-dimensionalized with the full wing area and the mean aerodynamic chord. The vortex breakdown (VBD) location is reported in non-dimensional terms with respect to root chord. In case of the wing/body, the wing-alone data have been obtained by subtracting the body-alone data from the wing/body data.

\section{Surface pressure model for delta wing}

Normal force and pitching moment coefficients of a delta wing are strongly dependent on the leeward flow. It is well-known that even at relatively low angles of attack the flow separates from both the leading edges and rolls up into two vortex sheets or cone-shaped cores of rotating fluid (Werle 1971). Flow attachment lines have been observed inboard of the vortex sheets and indicate that the air is drawn over the vortex sheets and accelerated downward. As the incidence is increased, the strength and location of the vortices over the wing change. They move higher and inboard with respect to the wing surface (Harvey 1961). Vertical movement is more pronounced than the inboard movement. At a particular angle of attack (depending on the wing-sweep angle), the vortices break down at the trailing edge. As the incidence is increased further, the vortex breakdown point moves towards the wing apex. When the breakdown point has reached the wing apex, the flow over the lee-side is completely separated (Werle 1971; Zohar \& Er-El 1988; Roos \& Kegelman 1990). At all angles of attack the leeside surface pressure is lower than the freestream pressure, while the lower surface pressure is above the freestream pressure. At sufficiently high angles of attack, the wing behaves as a flat plate aligned normal to the flow.

Figure 2 shows the normal force and pitching moment about wing apex for a $60^{\circ}$ delta wing (Zohar \& Er-El 1988). For this sweep angle, the VBD has been observed close to $14^{\circ}$ angle of attack on the trailing edge and close to $33^{\circ}$ at the wing apex. 

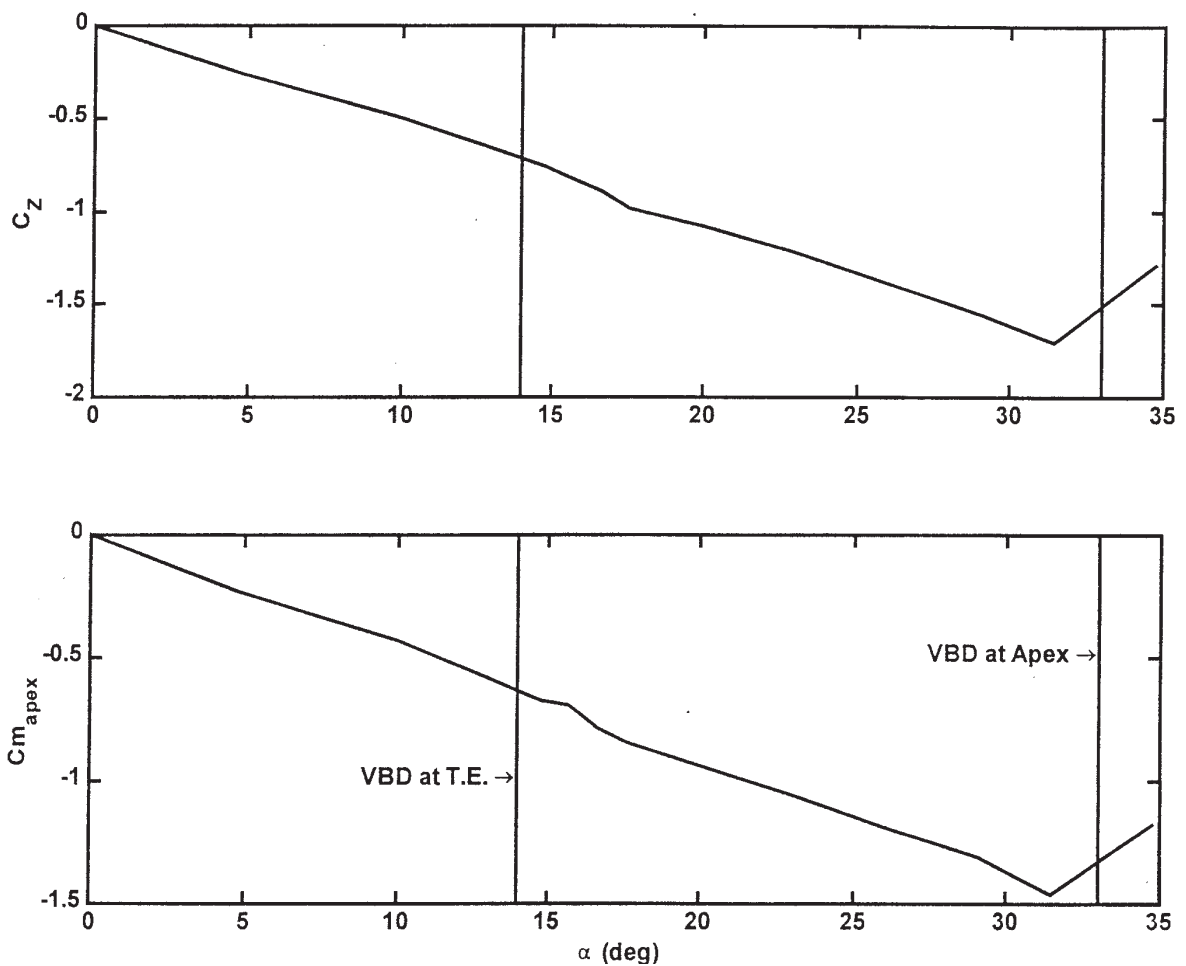

Figure 2. Normal force and pitching moment characteristics of a $60^{\circ}$ sweep delta wing (Zohar \& Er-EL 1988).

Figures $3 \mathrm{a}$ and $\mathrm{b}$ show typical pressure distributions for various angles of attack (Zohar $\&$ Er-El 1988). In figure 3a, the chordwise pressures are not along a particular radial or at a fixed spanwise coordinate. They are the measured peak pressures at particular chordwise locations and therefore are just below the vortex core. Also indicated in the figure is the VBD location. The following features are apparent from the pressure distributions.

(1) The spanwise pressure distribution shows a peak between 0.69 and 0.77 of the nondimensional local semi-span coordinate. The pressure distribution shows a clear pattern of increasing peak pressure with angle of attack, in agreement with the increasing strength of the leading edge vortex.

(2) As the VBD approaches the wing apex with increasing angle of attack, the spanwise pressure distribution tends to spread out, indicating the increased radius of the vortex core on the wing. Interestingly, the peak pressure (i.e., pressure below the vortex core) distribution from apex to trailing edge does not show any abrupt change due to progression of the VBD over the wing as angle of attack increases. However, the curves become steeper when vortex breakdown is over the wing. This is consistent with the spreading out of the vortex core behind the VBD location.

(3) At angles of attack beyond VBD at wing apex, the spanwise and chordwise pressure distributions collapse and become uniform. This occurs within a few degrees of wing apex breakdown angle of attack. 


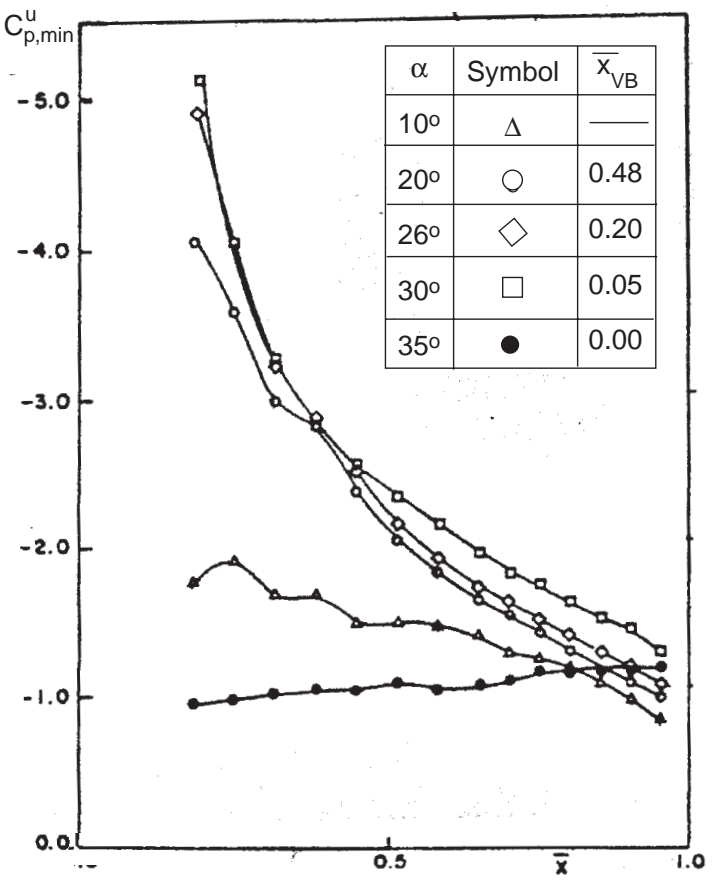

(a)

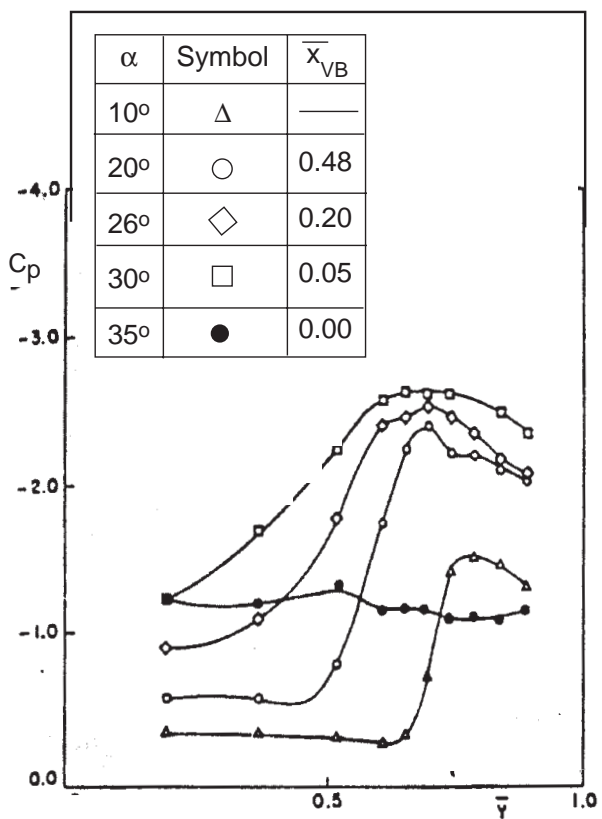

(b)

Figure 3. (a) Peak pressure distribution (below vorted core) and (b) spanwise pressure distribution at $45 \%$ of root chord on lee-side of a $60^{\circ}$ sweep delta wing $(\bar{x}=0.45)$ (Zohar \& Er-El 1988).

These observations are also valid for the $75^{\circ}$ delta wing reported by Zohar \& Er-El (1988). It is clear that if appropriate functions are chosen for the spanwise and chordwise pressure distributions, a basis for model structure determination can be obtained.

\subsection{Spanwise pressure coefficient model}

To begin with, for a fixed angle of attack, at any given chordwise location between the wing apex and the VBD position, a cubic variation is postulated for the spanwise pressure coefficient variation (figure 4).

$$
C_{p}(\eta)=(27 / 4) C_{P m}\left(\eta^{2}-\eta^{3}\right),
$$

where, $C_{P m}$ is the peak value of the pressure coefficient at the given chordwise location and $\eta(=y / s(x), s(x)$ being the local semi-span $)$ is the non-dimensional semi-span coordinate. The spanwise pressure coefficient along the entire span at locations between the VBD position and the wing trailing edge is chosen to be constant,

$$
C_{p}(\eta)=C_{P m} .
$$

This is in agreement with the collapse of the pressure distribution when VBD reaches the wing apex. At an angle of attack where the VBD is on the wing surface, after the VBD point, there is a gradual transition from the 'peaked' distribution to the 'flat' pressure distribution 


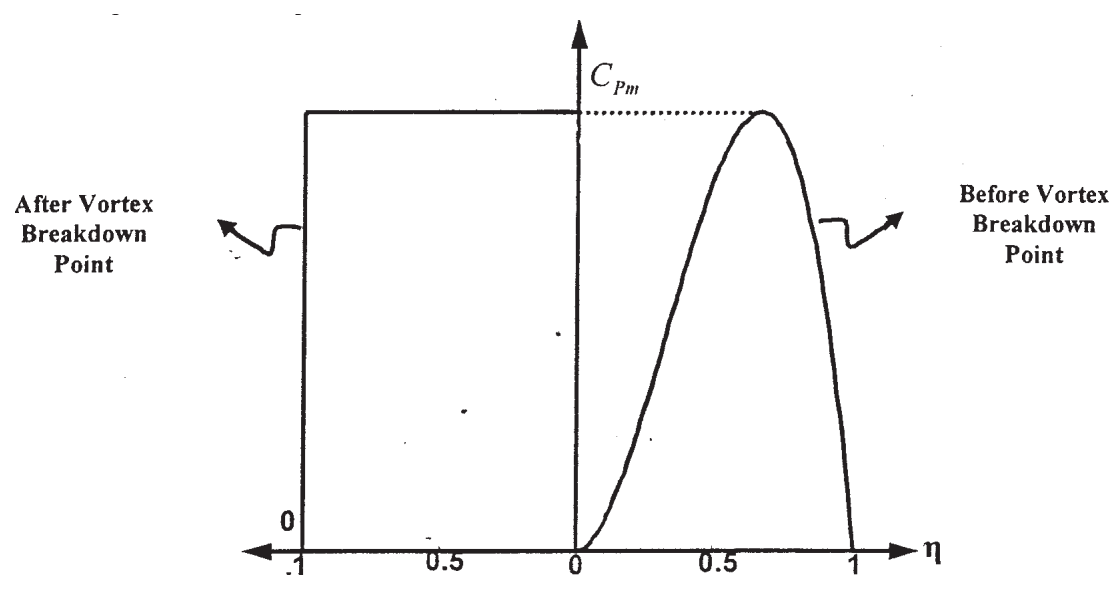

Figure 4. Spanwise variation of the pressure model.

behind VBD position. The abrupt change from (1) to (2) behind the VBD point is acceptable as a first approximation (Myatt \& Arena 1998). The spanwise distribution given by (1) has its peak value at $\eta=2 / 3$, which is quite close to the observed location in the experimental data. It must be noted that (1) assumes that the spanwise variation of pressure coefficients is independent of the chordwise position between wing apex and VBD position. The same is true for (2), behind the VBD position. This is equivalent to a conical flow assumption. Available experimental evidence supports this approximation (Roos \& Kegelman 1990, Zohar \& Er-El 1988).

\subsection{Development of chordwise peak pressure model}

The choice of a functional form for the chordwise peak pressure must fit the experimental values of figure 3a. It has been shown by Greenwell \& Wood (1992) that surface pressure measurements can be used effectively to estimate the chordwise vortex breakdown location by use of a shape parameter called the half width. In the development of this parameter, a simple point vortex over an infinite plate is used to derive some results. The surprisingly good correlation of the half width with experimental data, suggests that the point vortex over a flat plate may be a good first approximation to model the leeward surface pressure.

4.2. a Point vortex model: For a slender wing at low angles of attack, the flow can be assumed to be conical, and hence the problem of estimating the aerodynamic loads reduces to two-dimensional flow in a crossflow plane (Er-El et al 1989). The simplest possible model of the flow induced by a leading-edge vortex is a point vortex over an infinite plane due to Greenwell \& Wood (1992). The simple point vortex model gives rise to a pressure distribution quite similar to that seen on a delta wing. Greenwell \& Wood (1992) have shown that for this model the vortex strength $(\Gamma)$ is related to the vertical distance $(z)$ and the non-dimensional peak pressure coefficient $\left(C_{P m}\right)$ by the relation,

$$
\Gamma=\pi V_{\infty} z \sqrt{-C_{P m}},
$$


where $V_{\infty}$ is the freestream velocity. It is also known from the work of Hemsch \& Luckring (1990) and Visser \& Nelson (1993), that the trailing edge vortex strength for delta wings is given by

$$
\Gamma_{T . E .}=V_{\infty} c_{r} 4.63 \tan ^{0.8} \varepsilon \tan ^{1.2} \alpha \cos \alpha,
$$

where $\varepsilon$ is the semi-apex angle of the delta wing, $c_{r}$ is the root chord and a is the angle of attack. Assuming that the vorticity is generated linearly along the leading edge, the circulation at a distance $x$ along the root chord is given by

$$
\Gamma_{x}=V_{\infty} x 4.63 \tan ^{0.8} \varepsilon \tan ^{1.2} \alpha \cos \alpha .
$$

Equating this expression with the point vortex model given by (3) results in an expression for the vertical distance $z$ as a function of the chordwise coordinate $x$ and the peak pressure at that location

$$
z=\left(x 4.63 \tan ^{0.8} \varepsilon \tan ^{1.2} \alpha \cos \alpha\right) /\left(\pi \sqrt{-C_{P m}}\right) .
$$

The above expression can be rewritten in the non-dimensional form by dividing both sides by the root chord $c_{r}$. In this form, (6) has been used to estimate the vertical location of the vortex core above the $60^{\circ}$ delta wing from experimental data (Zohar \& Er-El 1988). The results are denoted in figure 5 a by an asterix $(*)$. The wing profile is shown as a solid line. Also shown in figure 5 is the result of using the following function to fit the peak pressure data (shown by a dotted line)

$$
C_{P m}(\xi)=\left(C_{P \max }-C_{P s}\right)(1-\xi) e^{a \xi}+C_{P s},
$$

where $\xi$ is the chordwise location from the wing apex non-dimensionalized against the root chord of the delta wing $\left(=x / c_{r}\right)$. It is seen that the function given by (7) essentially represents

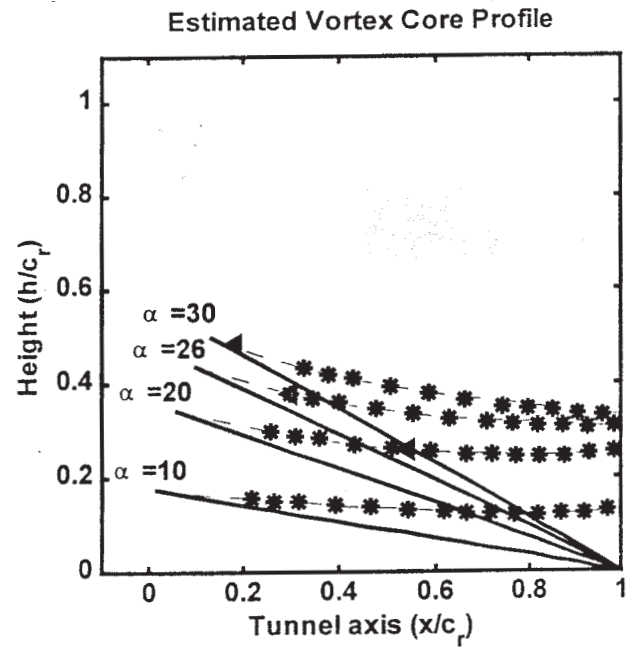

(a)

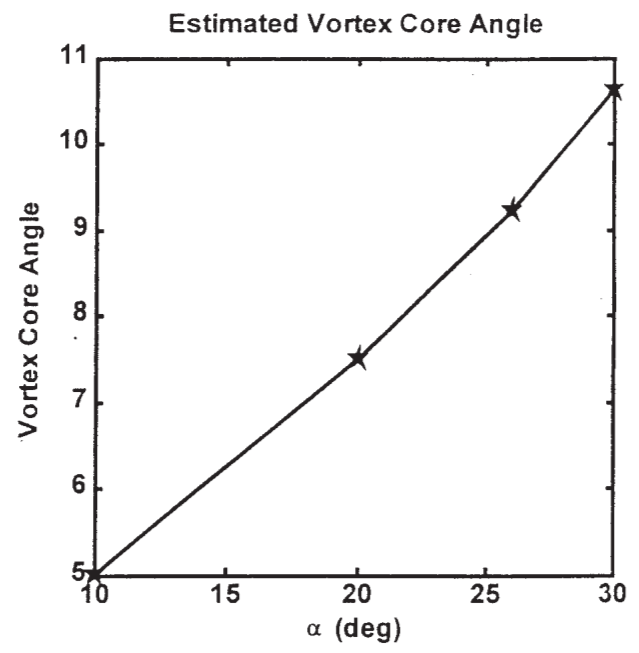

(b)

Figure 5. (a) Estimated vorted core profile above wing (* data, - model fit, $\delta$ VBD position), and (b) vorted core angle at wing apex at various angles-of-attack. 
the peak pressure below a vortex filament over the wing. The parameters $C_{P \max }$ and $C_{P s}$ represent the peak pressures at wing apex and trailing edge respectively. The parameter $a$ represents the curvature factor of the function. This comes into play when vortex breakdown is present on the wing surface. In figure $5 \mathrm{~b}$, the side elevation of the vortex core axis (the imaginary line that passes through the model apex and estimated vortex core location) is shown as a function of the angle of attack. This shows an approximately linear trend with angle of attack similar to that in the work of Guglieri \& Quagliotti (1997) and Hemsch (1989). Finally, the function given by (7) has the property that, when $C_{P \max }$ equals $C_{P s}$, the peak pressure distribution becomes constant. This property is desirable after the VBD reaches the wing apex.

\subsection{Leeward surface pressure model}

To summarize, the functional relations that describe the leeward surface pressure distribution are

$$
C_{P}(x, y)= \begin{cases}(27 / 4) C_{P m}(x)\left([y / s(x)]^{2}-[y / s(x)]^{3}\right), & x \leq x_{V B D}, \\ C_{P m}(x), & x>x_{V B D},\end{cases}
$$

where

$$
C_{P m}(x)=\left(C_{P \max }-C_{P s}\right)\left(1-x / c_{r}\right) e^{-a x / c_{r}}+C_{P_{S}} .
$$

$x_{V B D}$ is the location of the vortex breakdown point on the wing. The normal force can be obtained by integration of the function (8) over the delta wing surface,

$$
C_{\text {Zupper }}=-\frac{2}{S}=\int_{0}^{c_{r}} \int_{0}^{s(x)} C_{P}(x, y) \mathrm{d} x \mathrm{~d} y
$$

where $S$ is the wing area ( $=c_{r}^{2} \tan \varepsilon$, $\varepsilon$ being the wing semi-apex angle). In terms of the wing semi-apex angle, the local semi-span is given by $s(x)=x \tan \varepsilon$. The factor of two ahead of the integral is to account for the equal contributions from the two halves of the delta wing. The negative sign in front of the integral accounts for the positive upward sign convention for the suction pressure coefficient on the leeward surface.

A change of the independent variables $x$ and $y$ to the non-dimensional variables,

$$
\xi=x / c_{r} \text { and } \eta=y /(x \tan \varepsilon)
$$

results in the following integral,

$$
C_{\text {Zupper }}=-2 \int_{0}^{1} \xi \cdot C_{P m}(\xi)\left[\int_{0}^{1} f(\eta) \mathrm{d} \eta\right] \mathrm{d} \xi
$$

where

$$
f(\eta)= \begin{cases}(27) /(4)\left(\eta^{2}-\eta^{3}\right), & \xi \leq \tilde{x}, \\ 1, & \xi>\tilde{x}\end{cases}
$$


$\tilde{x}$ is the non-dimensional coordinate of the separation point $\left(=x_{V B D} / c_{r}\right)$. The integral $\int_{0}^{1} f \eta \mathrm{d} \eta$ is the proportion of the area under the spanwise peak value at any given chordwise location. A cubic variation, as assumed here before VBD point, results in a value of 9/16 for this integral. After the VBD point, the integral has a value of 1. Accordingly, (10) can be written as the sum of two integrals

$$
C_{\text {Zupper }}=-\frac{9}{8} \int_{0}^{\tilde{x}} \xi \cdot C_{P m}(\xi) \mathrm{d} \xi-2 \int_{\tilde{x}}^{1} \xi \cdot C_{P m}(\xi) \mathrm{d} \xi .
$$

In a similar manner, the integral expression for the pitching moment about wing apex results in

$$
\begin{aligned}
C_{\text {mupper }} & =-\frac{2}{S \cdot \bar{c}} \int_{0}^{c_{r}} x \int_{0}^{s(x)} C_{P}(x, y) \mathrm{d} x \mathrm{~d} y \\
& =-3 \int_{0}^{1} \xi^{2} \cdot C_{P m}(\xi)\left[\int_{0}^{1} f(\eta) \mathrm{d} \eta\right] \mathrm{d} \xi \\
& =-\frac{27}{16} \int_{0}^{\tilde{x}} \xi^{2} \cdot C_{P m}(\xi) \mathrm{d} \xi-3 \int_{\tilde{x}}^{1} \xi^{2} \cdot C_{P m}(\xi) \mathrm{d} \xi .
\end{aligned}
$$

Here the mean aerodynamic chord $\bar{c}$ is defined by $(2 / 3) c_{r}$. Given the peak chordwise distribution of (7), the normal force and pitching moment coefficient contributions from the leeward pressure distribution are

$$
\begin{aligned}
C_{\text {Zupper }}= & \frac{7}{8}\left(C_{P \max }-C_{P S}\right)\left(\frac{\tilde{x}^{2}}{a}+\frac{(2-a)}{a^{2}} \tilde{x}+\frac{(2-a)}{a^{3}}\right) e^{-a \tilde{x}}+\frac{7}{16} C_{P s} \tilde{x}^{2} \\
& -C_{P s}+\frac{9(2-a)}{8 a^{3}}\left(C_{P \max }-C_{P s}\right)-\frac{2}{e^{a}}\left(C_{P \max }-C_{P s}\right) \\
& \left(\frac{1}{a}+\frac{(2-a)}{a^{2}}+\frac{(2-a)}{a^{3}}\right) \\
C_{\text {mupper }}= & \frac{21}{16}\left(C_{P \max }-C_{P s}\right)\left(\frac{\tilde{x}^{3}}{a}+\frac{(3-a)}{a^{2}} \tilde{x}^{2}+\frac{2(3-a)}{a^{3}} \tilde{x}+\frac{2(3-a)}{a^{4}}\right) e^{-a \tilde{x}} \\
& +\frac{7}{16} C_{P s} \tilde{x}^{3}-C_{P s}-\frac{27(3-a)}{8 a^{4}}\left(C_{P \max }-C_{P s}\right) \\
& -\frac{3}{e^{a}}\left(C_{P \max }-C_{P s}\right)\left(\frac{1}{a}+\frac{(3-a)}{a^{2}}+\frac{2(3-a)}{a^{3}}+\frac{2(3-a)}{a^{4}}\right)
\end{aligned}
$$

It is interesting to examine the limiting case when the vortex breakdown point reaches the wing apex $(\tilde{x}=0)$ and the peak pressure over the leeward surface has collapsed $\left(C_{P \max }=C_{P s}\right)$. The expressions in (13) reduce to,

$$
C_{\text {Zupper }}=C_{\text {mupper }}=-C_{P s} .
$$

This is an important boundary condition at high angles of attack after VBD has completely destroyed the leeward vortices on the wing. It is a direct consequence of the choice of the 
function in (7). This aspect of the leeward surface pressure distribution is confirmed by Zohar \& Er-El (1988) and independently by Roos \& Kegelman (1990). Beyond the VBD point and after the pressure on the leeside has collapsed, the upper surface contribution to the normal force and the pitching moment must become equal to the uniform value of the residual suction on the upper surface. The experimental results reported in Zohar \& Er-El (1988) show that for the $60^{\circ}$ delta wing, the upper surface suction pressure collapses to values close to -1.0 . Compare this with the net normal force and pitching moment coefficient of figure 2. It is clear that the upper surface alone does not account for the normal force and pitching moment. The other contribution comes from the lower surface as was pointed out previously. This needs to be modelled if we have to account for the total force and moment.

\subsection{Windward surface model}

Zohar \& Er-El (1988) also present the lower surface pressure distribution of the 60-degree delta wing for various angles of attack. This is reproduced in figures $6 a$ and $b$. A few features of the lower surface pressure are noteworthy.

(1) Vortex breakdown does not have any appreciable effect on the lower surface pressure distribution.

(2) The spanwise distribution of pressure is seen to be practically constant, except at the edges where it is expected to go to zero. Therefore, parabolic variation is assumed.

The above observations also hold for the $70^{\circ}$ delta wing. Based on these observations the following lower surface pressure distribution is proposed for the $60^{\circ}$ delta wing

$$
C_{P}^{1}(x, y)=-C_{P m}^{1}(x)\left(1-[y / s(x)]^{2}\right),
$$

where $C_{P m}^{1}(x)=C_{P \max }^{1}\left(1-e^{5\left(x / c_{r}-1\right)}\right)$ and $C_{P \max }^{1}$ is the peak lower surface pressure at the centreline of the delta wing in the chordwise direction. The negative sign is introduced to account for the fact that although the lower surface pressure is positive, it gives rise to negative normal force and pitching moment. Suitable integration of the lower surface pressure distribution given by (15) over the planform results in the expressions for the lower surface contributions to the normal force and pitching moment coefficients,

$$
\begin{aligned}
& C_{\text {Zlower }}=-C_{p \max }^{1}(2 / 75)\left(17-2 e^{-5}\right), \\
& C_{\text {mlower }}=-C_{P \max }^{1}(2 / 375)\left(74+6 e^{-5}\right) .
\end{aligned}
$$

The chordwise peak pressure $C_{P \max }^{1}$ increase is approximately linear with angle of attack $(=K \alpha)$ as can be inferred from figure $6 \mathrm{a}$. The net result is that the lower surface contribution from (16) can be modelled as

$$
\begin{aligned}
C_{\text {Zlower }} & =-a_{1} \alpha, \\
C_{\text {mlower }} & =-a_{2} \alpha .
\end{aligned}
$$

This is the desired model structure for the lower surface contributions for a delta wing. The constants $a_{1}$ and $a_{2}$ can be obtained by satisfying (14) after the apex vortex breakdown angle of attack. In order to accomplish this, we first need the angle of attack for VBD at the apex. Then by trial and error one can choose the constants $a_{1}$ and $a_{2}$ to achieve collapse between normal force and pitching moment coefficients after apex breakdown angle of attack. 
(a)

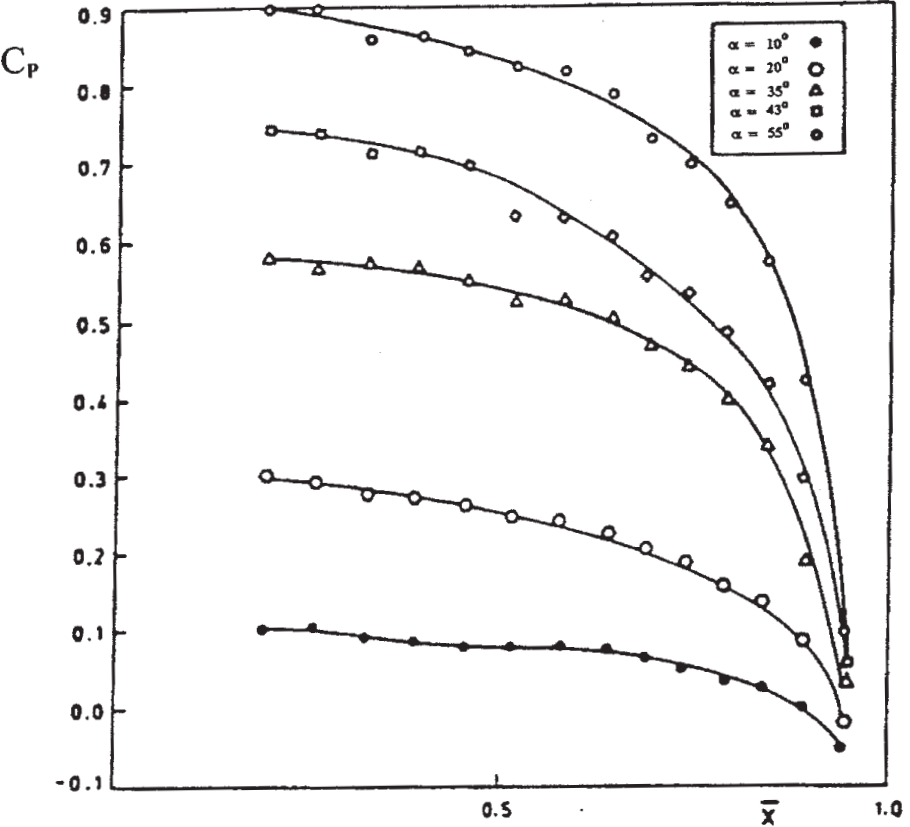

(b)

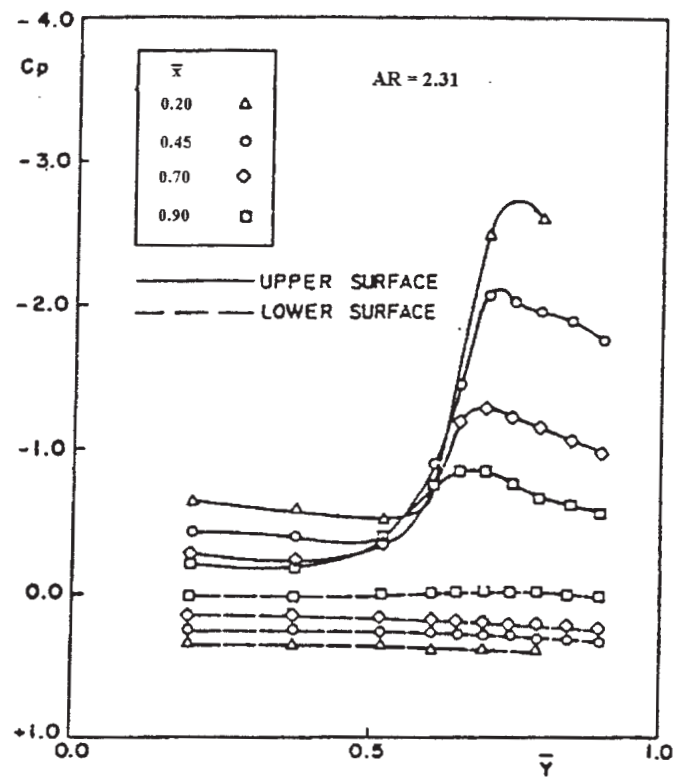

Figure 6. (a) Lower surface chordwise pressure profiles along $\bar{y}=0.2$ on the $60^{\circ}$ delta at various angles-of-attack (Zohar \& Er- El 1988). (b) Spanwise pressure distributions on a $60^{\circ}$ delta wing at $15^{\circ}$ angle-of-attack (Zohar \& Er-El 1988).

\subsection{The complete model structure}

It is well-known that at zero angle of attack, a thin wing generates no net lift or pitching moment. The delta wings experimental data used in this report with the exception of that of 
Vishwanath \& Patil (1992) have symmetric sharp leading edges. This gives rise to camber effects, which must be accounted for in the model structure. The model postulated above gives zero pitching moment when the normal force is zero. The camber effect is accounted for by subtracting the constant value of pitching moment coefficient at zero normal force coefficient (denoted by $\left.C_{m}\right|_{C_{z}=0}$ ) from the pitching moment data. In short, the total normal force and pitching moment coefficient model is

$$
\begin{aligned}
& C_{Z}=C_{\text {Zupper }}+C_{\text {Zlower }}, \\
& C_{m}=C_{\text {mupper }}+C_{\text {mlower }}+\left.C_{m}\right|_{C_{z}=0} .
\end{aligned}
$$

The wing/body data of Vishwanath \& Patil (1992) was also examined. The presence of the body can alter the overall characteristics by interaction. In particular, the fuselage vortices can affect the wing flow field. The wing/body interaction is relatively low up to $25^{\circ}$ angle of attack (Vishwanath \& Patil 1992). At high angles of attack, it is well-known that the vortex development is asymmetric. This asymmetry is primarily due to the long forebody. Since only the longitudinal characteristics are being modelled, this aspect is ignored. The additional effect of the body is discounted by augmenting the model with the contribution of the body alone experimental data of Vishwanath \& Patil (1992). Therefore, the model structure for the wing/body becomes

$$
\begin{aligned}
& C_{\text {ZWingbody }}=C_{\text {Zupper }}+C_{\text {Zlower }}+C_{\text {Zbody }}, \\
& C_{\text {mWingbody }}=C_{\text {mupper }}+C_{\text {mlower }}+C_{\text {mbody }}+\left.C_{m}\right|_{C_{z}=0} .
\end{aligned}
$$

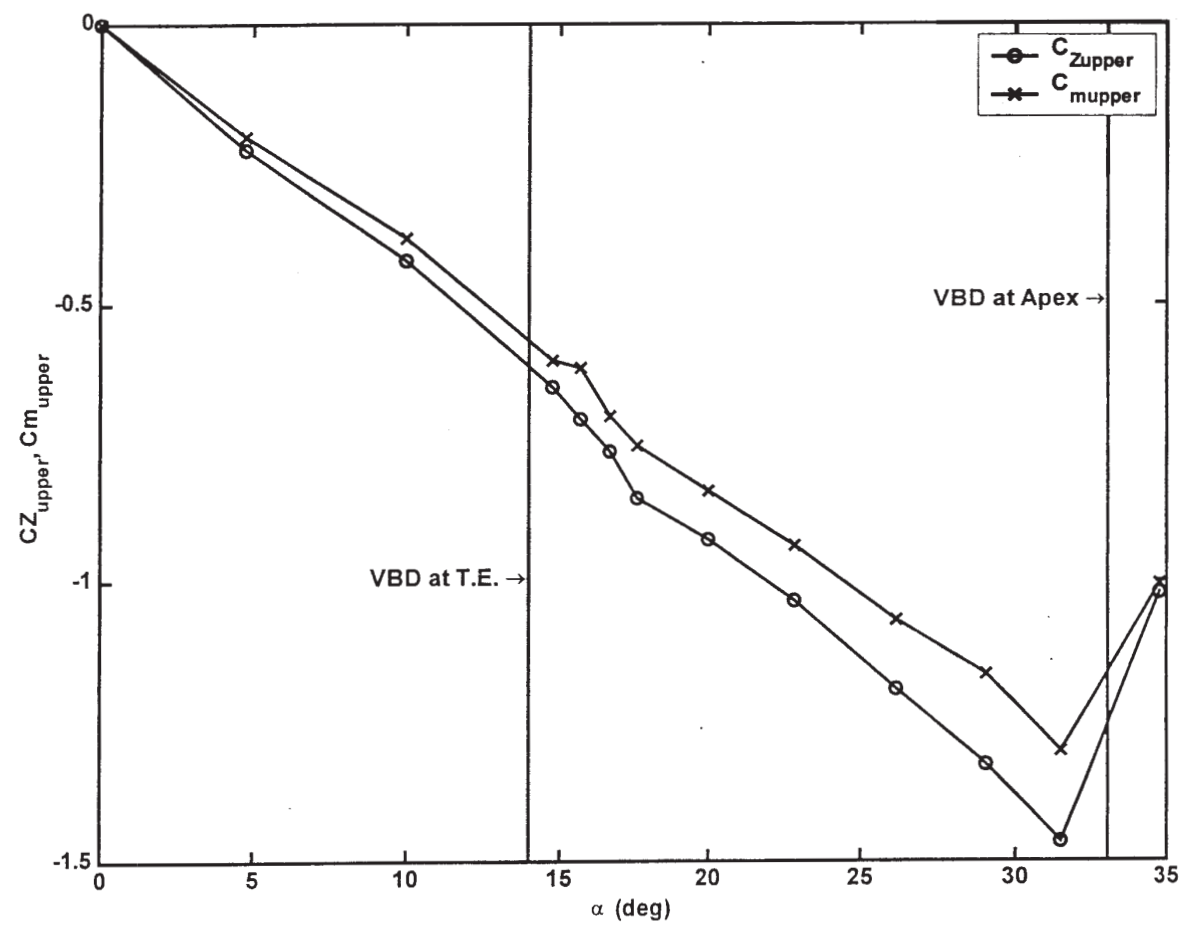

Figure 7. Upper surface contribution to normal force and pitching moment for the $60^{\circ}$ delta wing (Zohar \& Er-El 1988). 


\subsection{Application to data}

The above model was used to infer the contributions of the leeward surface to the normal force and pitching moment coefficients. Results for data from Zohar \& Er-El (1988) for the $60^{\circ}$ delta wing are presented in figure 7 . The lower surface pressure contribution was computed using (16) and $C_{P \max }^{1}$ was modelled as $K \alpha$, with value of $K=0.0171$ which was obtained from fgure 6 . The comparison clearly shows that within a few degrees of the leading edge vortex reaching the apex, the upper surface contribution to normal force and pitching moment coefficient collapse to the same value, as expected from (14). Further, this value is close to -1.0 which is the value reported for the same wing at about $32^{\circ}$ angle of attack (Zohar \& ErEl 1988). This clearly validates the modeling concept. A similar result was obtained for the $75^{\circ}$ wing data of Zohar \& Er-El (1988) in Pashilkar (2000). In the general case, (16) should be used to obtain the desired collapse in the upper surface contribution to normal force and pitching moment coefficients.

The above model was applied to data from Wentz \& Kohlman (1968) for the $60^{\circ}$ delta wing. The desired collapse is seen in the upper surface contribution in figure 8 . Two vertical lines on each graph show the breakdown region. A feature noticeable in figures 7 and 8 is that the collapse of the normal force and pitching moment coefficients takes place a few degrees after the observed apex VBD angle of attack, suggesting that the pressure collapse occurs a little after the VBD has reached the apex. The estimated values of the constants $a_{1}$ and $a_{2}$ obtained in this manner are tabulated in table 1 for a range of delta wing data. A perusal of the values

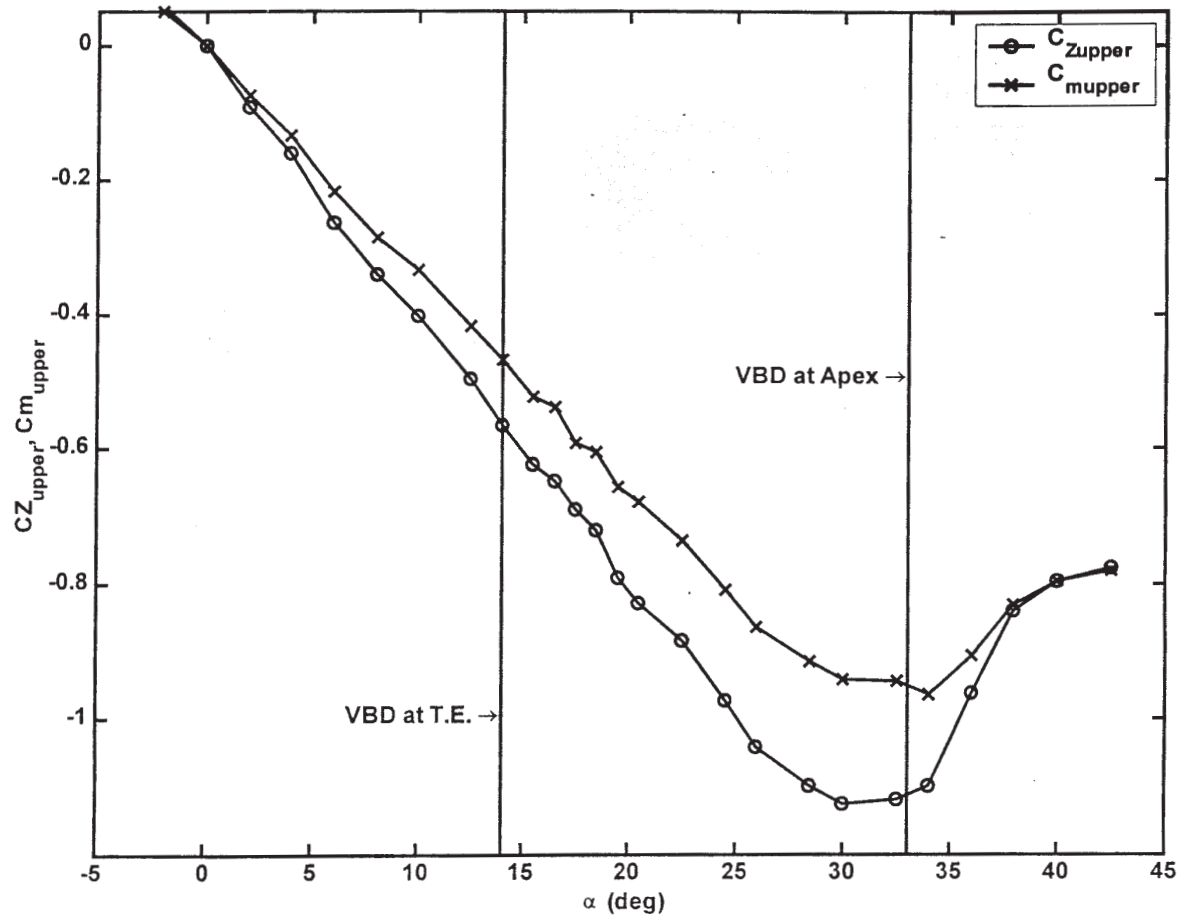

Figure 8. Upper surface contribution to normal force and pitching moment for the $60^{\circ}$ delta wing (Wentz \& Kohlman 1968). 
Table 1. Estimated lower surface model parameters for delta wings.

\begin{tabular}{|c|c|c|c|c|c|}
\hline \multicolumn{3}{|c|}{ Zohar \& Er-El (1988) } & \multicolumn{3}{|c|}{ Wentz \& Kohlman (1968) } \\
\hline $\begin{array}{l}\text { Sweep } \\
\text { (deg) }\end{array}$ & $a_{1}$ & $a_{2}$ & $\begin{array}{c}\text { Sweep } \\
\text { (deg) }\end{array}$ & $a_{1}$ & $a_{2}$ \\
\hline 60 & 0.0078 & 0.0051 & 50 & 0.0047 & 0.0013 \\
\hline \multirow[t]{2}{*}{75} & 0.0065 & 0.0050 & 60 & 0.0073 & 0.0022 \\
\hline & & & 70 & 0.0079 & 0.0038 \\
\hline
\end{tabular}

shows that within a given set of data, the values show a consistent pattern. However, for the $60^{\circ}$ delta wing, values obtained from one data set do not match the other. Another difference to be noted between figures 6 and 7 is that values in the former collapse close to -1.0 , whereas in the latter, they do so close to a value of -0.8 . This is the result of the inconsistency between the measurements of total normal force and pitching moment coefficients due to Wentz \& Kohlman (1968) and Zohar \& Er-El (1988). It is important to remember that the physical reality implied by (14), namely, the collapse of the leeward pressure peaks to a uniform pressure is consistent across many different experiments and therefore, the inconsistency in the values of constants $a_{1}$ and $a_{2}$ or the fact that the common value of coefficients is not the same for both cases is of limited concern for the model structure. The value of -0.8 agrees with the results of the wing/body reported in Vishwanath \& Patil (1992). Yet another feature of the upper surface pressure is that the minimum value of the normal force and pitching
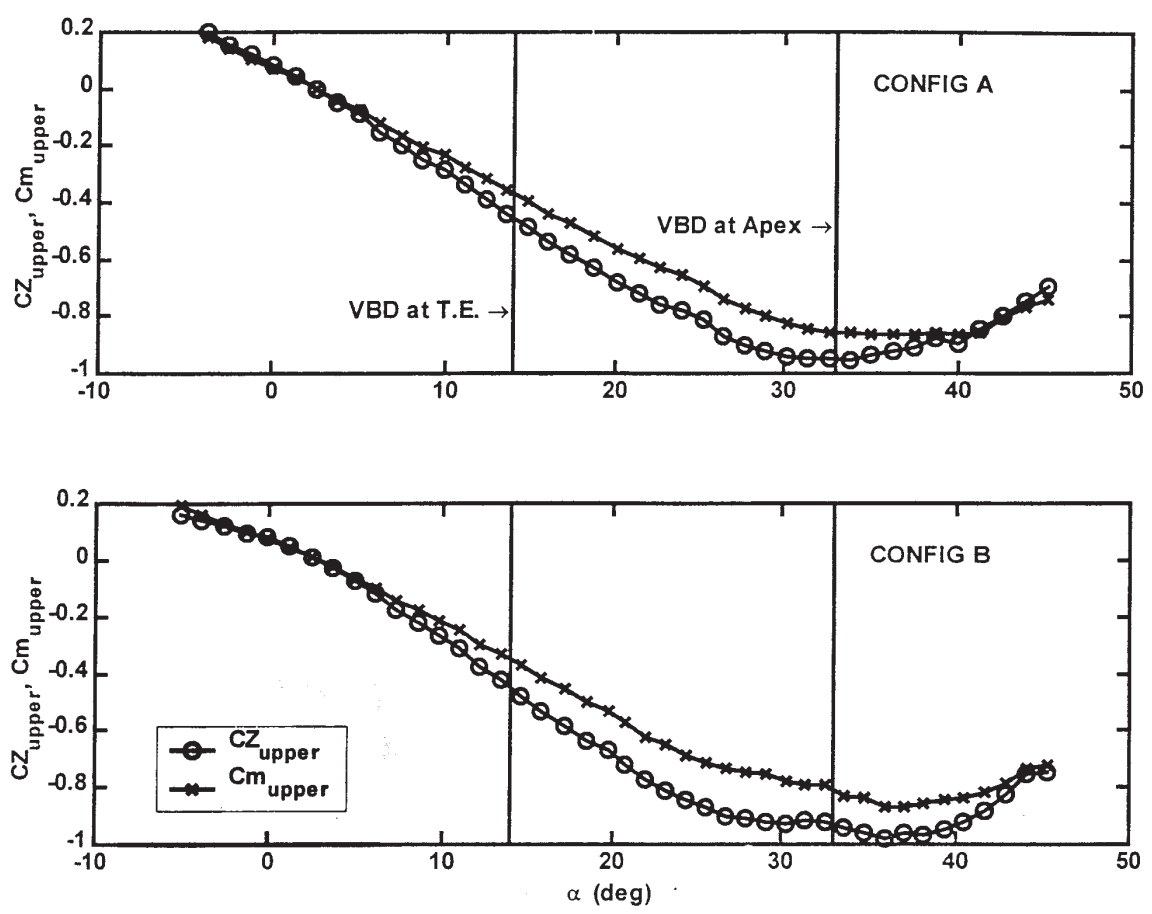

Figure 9. Upper surface contribution to normal force and pitching moment for the $60^{\circ}$ delta wing/body (Vishwanath \& Patil 1992). 
moment coefficients for the $50^{\circ}$ and $60^{\circ}$ delta wings is reached very close to the angle of attack at which vortex breakdown reaches the apex. This is not so for the $70^{\circ}$ delta wing, where the minimum value is reached somewhere between the angles of attack of the leading edge and the apex breakdown.

In figure 9, the upper surface contribution for the wing/body data of Vishwanath \& Patil (1992) is presented for the two configurations tested with $60^{\circ}$ delta wing. The pitching moment and normal force have been obtained by discounting the contribution of the body and camber effects. The lower surface contribution used was the same as that obtained for the data of Wentz \& Kohlman (1968) (table 1). The slenderness ratio of configuration A is 7.8 and that of B is 9.8. Examination of figure 9 reveals that the upper pressure contribution does collapse as expected (the vertical lines in these figures indicate the VBD at trailing edge and wing apex respectively). Similar collapse was verified for the rest of the data in Pashilkar (2000). The value towards which the normal force and moment coefficients tend to is close to -0.8 , which is indeed the value also seen for the data of Vishwanath \& Patil (1992). The minimum value of normal force and pitching moment coefficients is reached close to the apex breakdown in the case of the $60^{\circ}$ delta wing. It is clear that the proposed model is simple and effective.

\section{Model parameter estimation}

In the previous section, it has been shown that the pressure model is consistent across a range of delta wings and wing/body combinations. The upper surface pressure model parameters now need to be estimated based on the data extracted above. The relations given by (13) for the upper surface normal force and pitching moment contributions are applicable at each angle of attack. This means that the functions $C_{P \max }(\alpha), C_{P S}(\alpha)$ and $\tilde{x}(\alpha)$ need to be determined.

\subsection{Vortex breakdown function $\tilde{x}(\alpha)$}

Wentz \& Kohlman (1968) have obtained vortex breakdown location data for (i.e., function $\tilde{x}(\alpha))$ for the delta wings over a range of sweep angles from $45^{\circ}$ to $80^{\circ}$. Traub (1996) has developed a simple functional fit to the vortex breakdown data as a function of the sweep and angle of attack. Stephen (1995) has examined data for rolled delta wing with respect to sweep and angle of attack and shown that a simple function fit allows one to collapse available vortex breakdown data for different sweep, angle of attack and sideslip. In this paper, the data of Wentz \& Kohlman (1968) have been taken as the reference for function $\tilde{x}(\alpha)$ in case of the pure delta wing data.

Experimental evidence (Straka \& Hemsch 1994) suggests that the body has a significant effect on the vortex breakdown location in a wing/body combination. In case of the wing/body data of Vishwanath \& Patil (1992), the breakdown location is not available. It has been found by Hummel \& Srinivasan (1967) that the slopes of the lift, drag and the pitching moment show significant change near the angle of attack at which vortex breakdown takes place. In figure 10 , the slopes of the lift and pitching moment are plotted as functions of the angle of attack for the wing/body data with $60^{\circ}$ wing sweep. It is seen that close to $15^{\circ}$, there is change in slope. This is used to fix the onset of trailing edge breakdown. It is assumed that the angle of attack for apex breakdown is not significantly affected by the presence of the body and therefore the corresponding delta wing value is used for the wing/body. A simple cubic function,

$$
\tilde{x}(\alpha)=\left(-2 \alpha^{3}+3\left(\alpha_{1}+\alpha_{2}\right) \alpha^{2}-6 \alpha_{1} \alpha_{2} \alpha+\left(3 \alpha_{1}-\alpha_{2}\right) \alpha_{2}^{2}\right) /\left(\alpha_{1}-\alpha_{2}\right)^{3},
$$




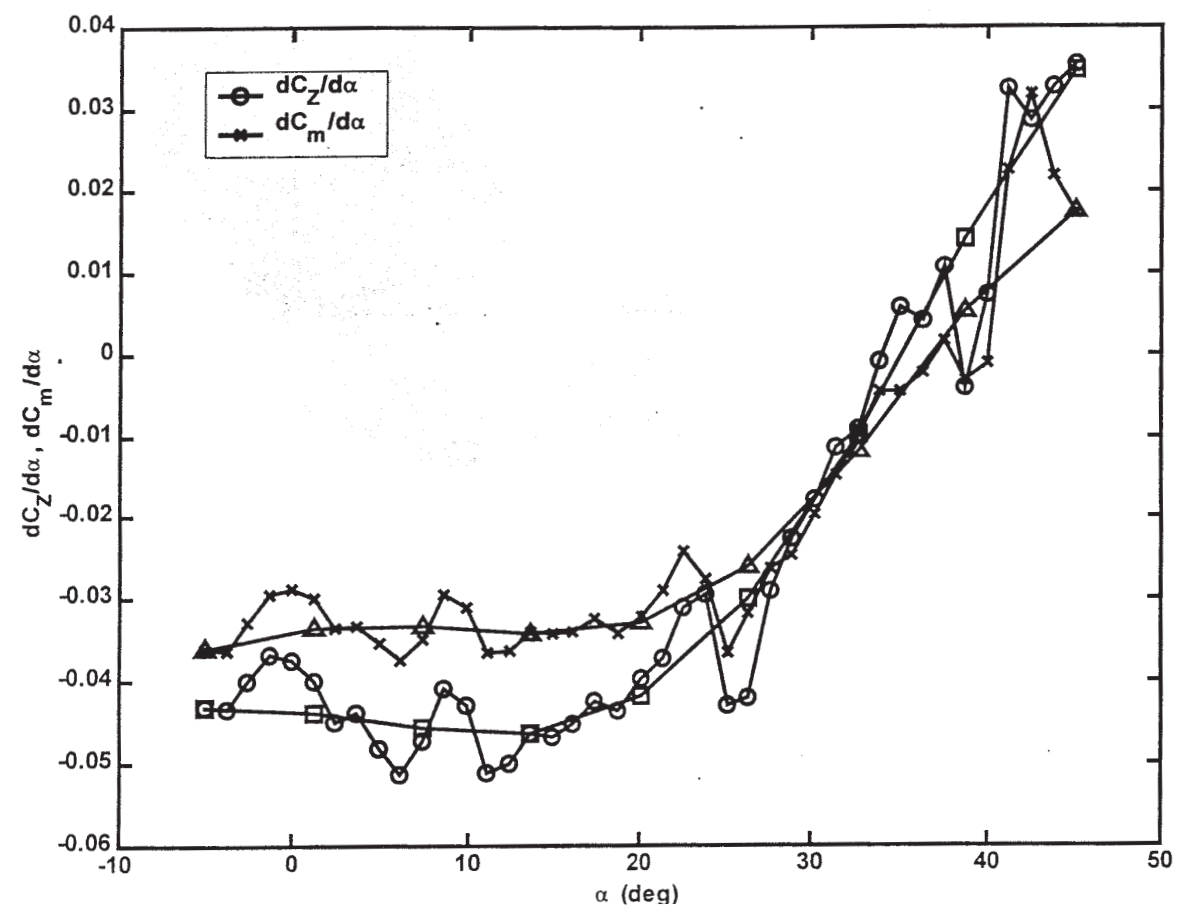

Figure 10. Static derivatives with angle-of-attack for the $60^{\circ}$ delta wing/body config A (Vishwanath $\&$ Patil 1992). Open triangles and squares show smoothed slopes.

where $\alpha_{1}$ and $\alpha_{2}$ are the angles of attack for trailing edge and leading edge breakdown, is used with the wing/body data of Vishwanath \& Patil (1992). It is clear that lift or pitching moment data are required before hand in order to infer the function $\tilde{x}(\alpha)$ for the wing/body. Since, this modelling method is based on the a priori requirement of such data from wind tunnel or flight test, this requirement does not pose any additional problems.

\subsection{The functions $C_{P S}(\alpha)$ and $a(\alpha)$}

Further simplification is achieved if the function $C_{P_{S}}(\alpha)$ is assumed to be linear in angle of attack to the point where it collapses and becomes equal to $C_{p \max }(\alpha)$. This is a reasonable simplification because the results are not as sensitive to the nature of variation of this function. The curvature factor $a$ is chosen to vary with the breakdown location. This is because, as pointed out earlier, experimental observations show that the peak pressure curve becomes steeper when breakdown is on the wing surface. The curvature factor is unity when $\tilde{x}=1$ and is linearly varied with $\tilde{x}$ to a value of 5 at $\tilde{x}=0$.

\subsection{The cost function}

The estimation process now involves finding a function $C_{P \max }(\alpha)$, such that the best possible fit to the static data is obtained given the known function $\tilde{x}(\alpha)$ and a linear variation in $C_{P s}(\alpha)$. 
The cost function for estimation is the least squares error between the upper surface data and the model for the angle of attack range,

$$
e=\sum_{a}\left[\left(y_{C Z}-C_{Z}\right)^{2}+\left(y_{C m}-C_{m}\right)^{2}\right]^{1 / 2},
$$

where $y_{C z}$ and $y_{C m}$ are the experimental normal force and pitching moment data and $C_{z}$ and $C_{m}$ are the respective values from the model. Since the function $C_{P \max }(\alpha)$ has to be estimated, the complete angle of attack range is divided into a number of break points and the values of the function at these points are the unknowns to be determined by minimizing the error given by (21).

\section{Results}

Figure 11 presents the results of the parameter estimation for $60^{\circ}$ delta wing data of Wentz $\&$ Kohlman (1968). The overall fit of the data is good and the model is capable of capturing the normal force and pitching moment characteristics of delta wings. The value of the peak pressure $C_{P \text { max }}$ is close to that reported by Zohar \& Er-El (1988). Another feature is that the peak pressure value $C_{P \max }$, reaches a maximum just near the angle of attack for vortex breakdown at wing apex. This is in agreement with the experimentally observed fact that the maximum is reached a few degrees short of the angle of attack for vortex breakdown at trailing edge.

The results for the wing/body data of Vishwanath \& Patil (1992) (configuration A) are shown in figure 12. Here the data represent wing-alone component obtained by subtracting the body-alone contribution from the total wing/body data. The results show that the estimated parameters are consistent with the values obtained from data of wing alone (Figure 11). This validates the model structure used for the wing/body case. It was also seen that the model
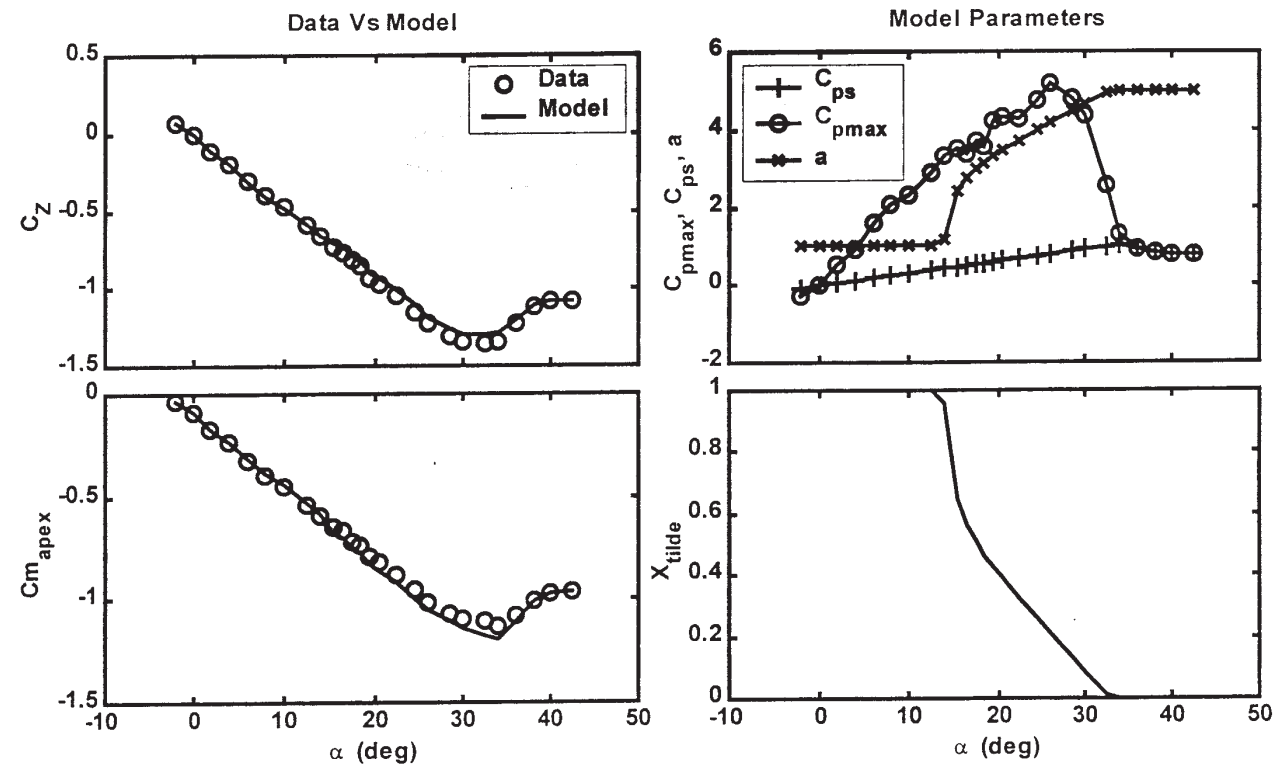

Figure 11. Model (solid line) against the data of Wentz \& Kohlman (1968) for a $60^{\circ}$ delta wing. 

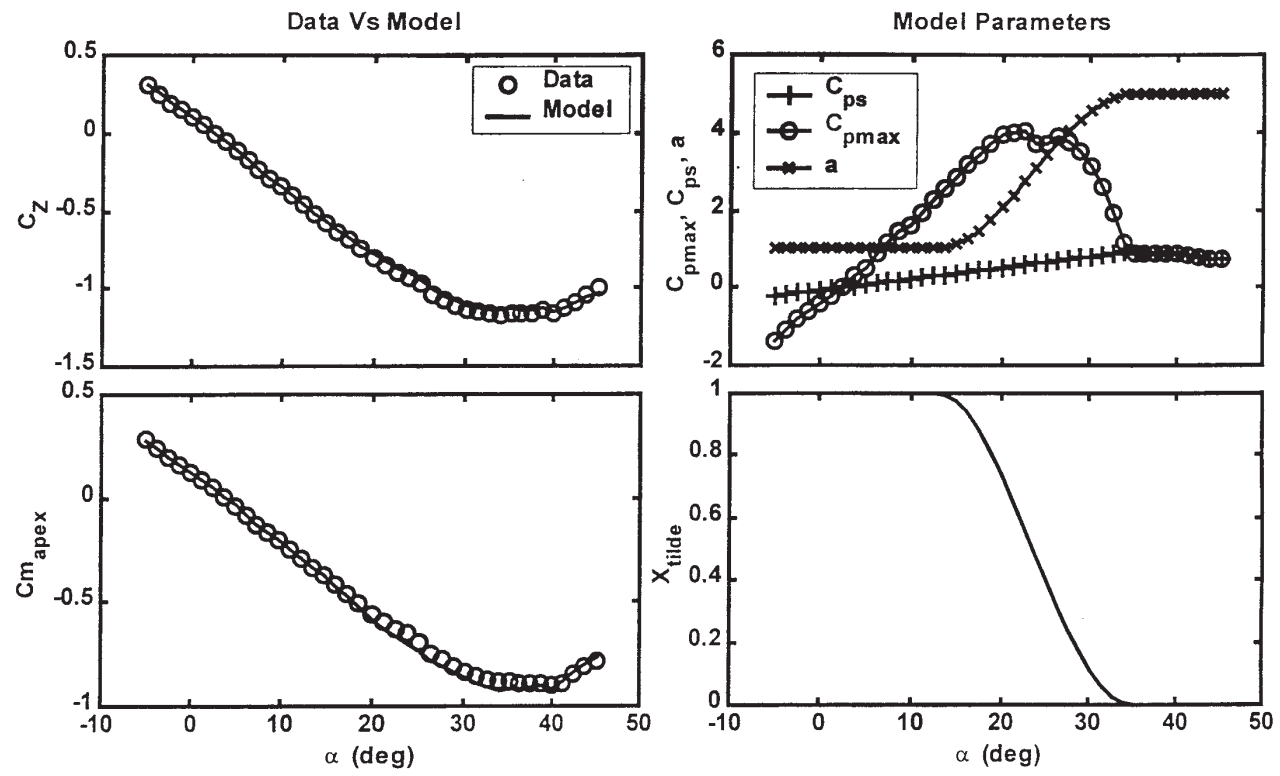

Figure 12. Model (solid line) against the data of Vishwanath \& Patil (1992) for a $60^{\circ}$ delta wing Config A.

gives a slightly better fit for configuration A which has a slenderness ratio of 7.8. This is expected considering the higher vortex interaction of the body with the wing for the more slender configuration $B(l / d=9.8)$ (figure 13). Matches with the data for $50^{\circ}$ and $70^{\circ}$ sweep
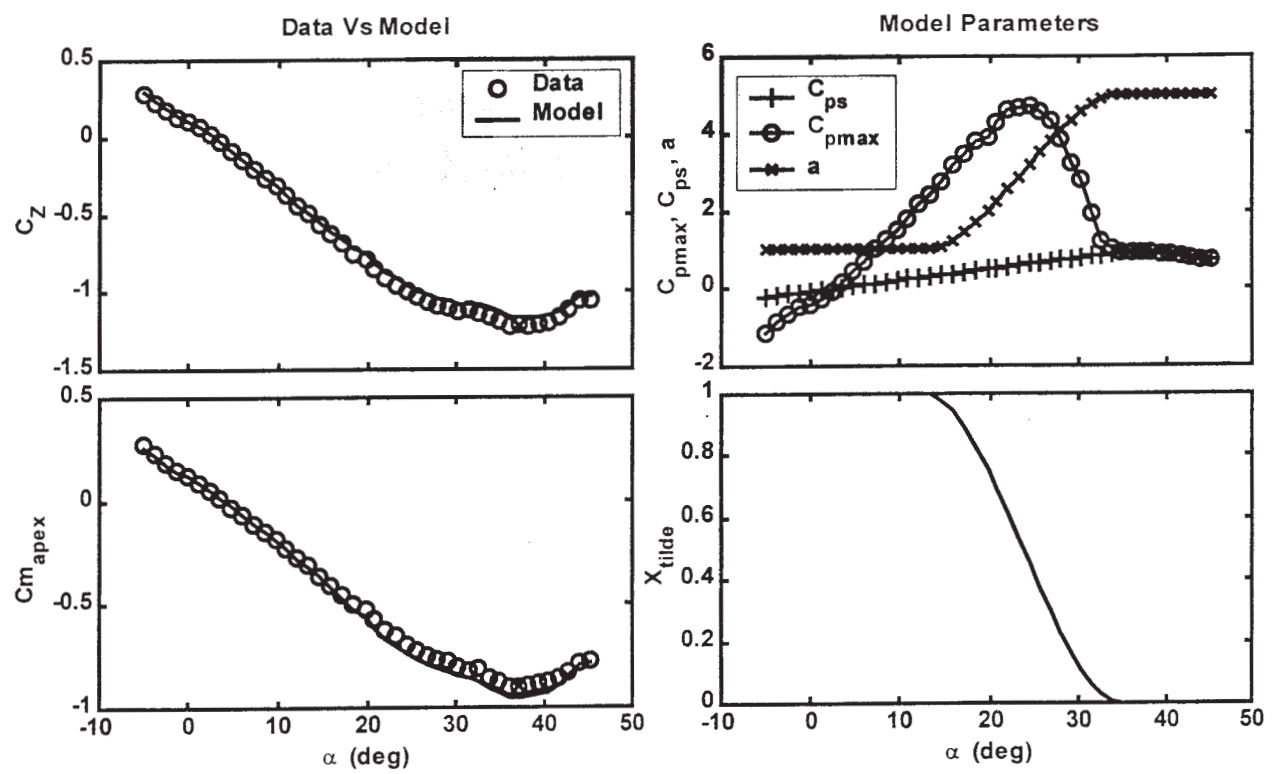

Figure 13. Model (solid line) against the data of Vishwanath \& Patil (1992) for a $60^{\circ}$ delta wing Config B. 
also show the same trends (Pashilkar 2000). By tuning the lower surface parameters $a_{1}$ and $a_{2}$, a better fit can be obtained for the wing/body data.

\section{Conclusions}

A new modelling structure based on the surface pressure distribution has been developed for simple delta wings and extended to wing/body longitudinal characteristics throughout the angle of attack range.

- The modelling structure is seen to be simple and physical. A good match has been shown with the experimental data for a $60^{\circ}$ delta wing and wing/body combination. The estimated parameters show close, though not exact values, as compared with the experimental data. The physical nature of the model ensures that all the estimated parameters are plausible.

- The initial results of the model are encouraging, indicating that there is scope to further improve the model. Extension to model unsteady aerodynamic pressures is a possibility.

The author is grateful to Dr P R Vishwanath and to Dr S Pradeep, Indian Institute of Science, for helpful discussions.

\section{References}

Er-El J, Seter D, Weihs D 1989 Nonlinear aerodynamics of delta wing in combined pitch and roll. J. Aircraft 26: 254-259

Greenwell D I, Wood N J 1992 Determination of vortex burst location on delta wings from surface pressure measurements. AIAA J. 30: 2736-2739

Guglieri G, Quagliotti F B 1997 Experimental investigation of vortex dynamics on a $65^{\circ}$ delta wing in sideslip. Aeronaut. J. 101: 111-120

Hancock G J, Lam J S Y 1987 Axiomatic modeling - Parts I to IV. Aeronaut. J. 91: 1-28, 72-88

Harvey J K 1961 Some measurements on a yawed slender delta wing with leading-edge separation. ARC R \& M No. 3160, Her Majesty's Stationery Office, London, UK

Hemsch M J 1989 Similarity for high-angle of attack subsonic/transonic slender-body aerodynamics. J. Aircraft 26: 56-66

Hemsch M J, Luckring J M 1990 Connection between leading edge sweep, vortex lift, and vortex strength for delta wings. J. Aircraft 27: 473-475

Hummel D, Srinivasan P S 1967 Vortex breakdown effects on the low-speed aerodynamic characteristics of slender delta wings in symmetric flow. J. R. Aeronaut. Soci. 71: 319-322

Jaramillo P T 1994 A multi-point model for the analysis of aircraft motion in complex flow fields. Ph D thesis, Wichita State University, Kansas City, KS

Klein V, Batterson J G 1983 Determination of airplane model structure from flight data using spline and stepwise regression. NASA TP-2126

Lieshmann J G, Beddoes J S 1989 A semi-empirical model for dynamic stall. J.Am. Helicopter Soc. 34: $3-17$

Morelli E A 1995 Global nonlinear aerodynamic modeling using multivariate orthogonal functions. J. Aircraft 32: 270-277

Myatt J H, Arena A S Jr 1998 A theoretical/empirical model for rolling delta wings with vortex breakdown. AIAA Paper $98-2527$

Pashilkar A A 2000 Modeling of static longitudinal aerodynamic data. NAL PD FC 0001

Polhamus E C 1966 A concept of the vortex lift of sharp-edge delta wings based on a leadingedgesuction_analogy. NASA TN D-3767 
Purvis J W 1981 Analytical prediction of vortex lift. J. Aircraft 18: 225-230

Raol J R, Jategaonkar R V 1995 Aircraft parameter estimation using recurrent neural networks- A critical appraisal. AIAA Paper 95-3504

Roos F W, Kegelman J T 1990 An experimental investigation of sweep-angle influence on delta-wing flows. AIAA Paper 90-0383

Shamroth S J 1985 Calculation of steady and unsteady airfoil flow-fields via the Navier- Stokes equations. NASA CR-3899

Straka W A, Hemsch M J 1994 Effect of fuselage on delta wing vortex breakdown. J. Aircraft 31: $1002-1005$

Stephen E J 1995 Analysis of rolled delta wing flows using effective sweep and attack angles. $J$. Aircraft 32: 978-984

Tobak M, Schiff L B 1976 On the formulation of aerodynamic characteristics in aircraft dynamics. NACA TR R-456

Traub L W 1997 Prediction of vortex breakdown and longitudinal characteristics of swept slender planforms. J. Aircraft 34: 353-359

Traub L W 1996 Simple prediction method for location of vortex breakdown on delta wings. J. Aircraft 33: 452-454

Tuncer I, Wu J, Wang C 1989 Theoretical and numerical studies of oscillating airfoils. AIAA Paper 89-0021

Vishwanath P R, Patil S R 1992 Aerodynamic characteristics of delta wing-body combinations at high angles of attack. NAL PD EA 9205

Visser K D, Nelson R C 1993 Measurements of circulation and vorticity in the leading-edge vortex of a delta wing. AIAA J. 31: 104-111

Wentz W H Jr, Kohlman D L 1968 Wind tunnel investigations of vortex breakdown on slender sharpedged wings. NASA CR 98737

Werle H 1971 Sur L’Éclatement Des Tourbillons. ONERA Note Technique $\mathrm{N}^{\circ} 175$

Zagainov G I 1993 High maneuverability. Theory and practice. AIAA Paper 93-4737

Zohar Y, Er-El J 1988 Influence of the aspect ratio on the aerodynamics of the delta wing at high angle of attack. J.Aircraft 25: 200-205 